\title{
Protective Effect of Cocoa Extract on Malondialdehyde Level in Ultraviolet B Induced - Albino Mice Skin
}

\author{
Suci Nugraeni Tahir ${ }^{1}$, Farida Tabri ${ }^{1}$, Khairuddin Djawad ${ }^{1}$, Anni Adriani $^{1}$, Arifin Seweng ${ }^{2}$, \\ Nasrum Massi ${ }^{3}$ \\ ${ }^{1}$ Departement Dermatology and Venereal, Faculty of Medicine, University of Hasanuddin, Makassar, Indonesia \\ ${ }^{2}$ Departement of Biostatistic, Faculty of Medicine, University of Hasanuddin, Makassar, Indonesia \\ ${ }^{3}$ Departement of Microbiology, Faculty of Medicine, University of Hasanuddin, Makassar, Indonesia
}

Email address:

ucinugraeni@mail.com (S. N. Tahir)

\section{To cite this article:}

Suci Nugraeni Tahir, Farida Tabri, Khairuddin Djawad, Anni Adriani, Arifin Seweng, Nasrum Massi. Protective Effect of Cocoa Extract on Malondialdehyde Level in Ultraviolet B Induced - Albino Mice Skin. American Journal of Clinical and Experimental Medicine.

Vol. 5, No. 2, 2017, pp. 41-45. doi: 10.11648/j.ajcem.20170502.13

Received: January 27, 2017; Accepted: February 10, 2017; Published: March 1, 2017

\begin{abstract}
The antioxidant activity in cocoa linked to the polyphenol content therein, especially subunit monomers catechin and epicatechin. The aim of the study was to determine the protective effects of cocoa extract on levels of malondialdehyde in albino mice by exposure to UVB. This study was conducted at animal laboratory to interventions UVB and Extract, Cocoa and Biomolecular laboratory of medical faculty of Hasanuddin University for the ELISA examination. This study used true experimental design with animal experimental design to assess the effectiveness of topical application cacao extract on mice skin after UVB induction for twelve weeks. The research samples were 30 head of albino mice divided into 6 groups: control 5 mice without protection and given $450 \mathrm{~mJ}$ UV Bexposure 3 times per week, the second group was 5 untreated controlmice, the third group was 5 mice with 100 ppm of cocoa topical extract every day and UVB $450 \mathrm{~mJ}$ exposure 3 times a week, the fourth group of 5 mice with $200 \mathrm{ppm}$ topical cocoa extract every day and exposure to $450 \mathrm{~mJ}$ three times a week, the fifth group of 5 mice with $400 \mathrm{ppm}$ topical cocoa extract every day and exposure to $450 \mathrm{~mJ}$ three times a week, the sixth group of 5 mice with $800 \mathrm{ppm}$ topical everyday cocoa extract and exposure to $450 \mathrm{~mJ}$ three times a week, a week after termination and biopsy excisionof skin for examination of Malondialdehyde level with ELISA. The results indicated resources of 200 ppm cocoa extract Provides the best effect with reduced MDA levels with the highest protective effect Compared to other groups.
\end{abstract}

Keywords: Cocoa Extract, Malondialdehyde, Ultraviolet B

\section{Introduction}

Cacao is produced from seeds of the cocoa plant (Theobroma cacao), which originate in the rain forests of South America and Central America. [1] Many studies confirm that consuming cocoa has benefits, health especially for cocoa contains flavonoids and rich in antioxidants. Benefits cocoa for health include reducing the risk of chronic diseases such as disease, cardiovascular cancer, and other diseases associated with age. [2, 3]

The antioxidant activity in cocoa linked to the polyphenol content there in, especially subunit monomers catechin and epicatechin. The concentration of flavanols final and final product antioxidant capacity of foods derived from cocoa depending on the type and origin of the cocoa beans, growing conditions, storage or post-harvest treatment and of processing products. Cocoa beans freshly harvested from the tree contains a lot of polyphenols and has antioxidant capacity is higher than food sources other such as green tea, pomegranate, gojiberry, and blueberry. [4]

Oxidative stress is a state of imbalance between free radicals with antioxidants, where the number of free radicals more when compared with antioxidants. If the production of free radicals exceeds the antioxidant ability to intracellular neutralize the excess free radicals potentially cause cell damage. This damage is often referred to as oxidative damage, that damage biomolecules constituent cells caused by reactions with free radicals. 
An increase in oxidative stress have a negative impact on some components the of cell membrane, which is damage to the lipid membrane forming Malondialdehyde (MDA), damage proteins, carbohydrates, and DNA. According to, damage the oxidative caused by free radicals implicated in various pathological conditions, which damage cells, tissues, and organs such as the liver, kidneys, heart, both humans and animals. This damage can end up in cell death resulting in the acceleration of the onset of degenerative diseases. In connection with the potential toxicity of free radicals, the body has a natural defense system mechanisms in the form of antioxidant enzymes endogenous that function and accelerate the degradation of compounds neutralize free radicals to prevent damage to components of the cell macromolecules. [5] The system is divided into two major groups, namely: preventive defense systems such as the enzyme superoxide dismutase, catalase, and glutathione peroxidase and defense systems through termination radical reactions such as isoflavones, vitamin A, vitamin C, and vitamin E. The body has three intracellular antioxidant enzymes or endogenous antioxidant, namely superoxide dismutase (SOD), glutathione peroxidase (GPx) and catalase (Cat). SOD is one of the antioxidants endogenous that serve to catalyze dismutase free radical superoxide anion (O2-) into hydrogen peroxide and oxygen molecules. [5]

Antioxidants are chemical compounds that can donate one or more electrons to free radicals, so that these free radicals can be muted. Free radicals are reactive molecules that have unpaired electrons. Various types of radicals free have the potential to interact with biological molecules in the body, such as proteins, lipids, and DNA, and trigger reactions that can damage and cause cell death. Various evidence that exist today have shown that exposure to free radicals involved in the etiology of various diseases. [6]

To measure the oxidative stress of the body can be determined by one of its parameters, namely Malondialdehyde (MDA). MDA is one of the indicators most commonly used as an indication of lipid peroxidation. MDA is a compound that can describe the activity of free radicals in cells that serve as a guide to oxidative stress caused by free radicals. [7]

Previous research by Djawad et al (2008), have shown a protective effect of curcumin on the expression of photoproduct and hyperplasia of the epidermis of mice. [8] Waspodo et al doing experimental research cacao extract in mice that are exposed UVB TGF- $\beta$ associated with an increase and decrease in MMP1 which marks an improvement on the network. [9]

Another study by Adriani et al, proving cocoa extract has a effect protective against ultraviolet radiation by reducing the expression of 8-OHdG, PCNA expression and hyperplasia. Research Wahab et al (2014), examines the topical application of extracts of cocoa before exposure to DMBA / TPA on albino mice have a protective effect against skin tumors through the effects of inhibition of oxidative stress and Bcl-2 in the initiation phase and induction of oxidative stress and Bcl-2 in phase promotion. [10]
Based on previous studies of cocoa have anti-oxidant and one of the parameters to investigate these effects is to measure the levels of Malondialdehyde (MDA). Thus this study aims to determine the protective effects of cocoa extract on levels of malondialdehyde in albino mice by exposure to UVB.

\section{Materials and Methods}

\subsection{Location and Time Research}

Conducted at the Laboratory Animal Research to Interventions UVB and Extract Cocoa Laboratory of Biochemical, Faculty of Medicine, University of Hasanuddin Makassar as the ELISA examination.

\subsection{Design and Research Variables}

This study is an experimental animal with designs. a purely experimental The research variables consist of: the independent variable (extract cocoa), variable the dependent (MDA), intermediate variable (UVB rays, oxidative stress), and the control variables (albino mice (age, sex, food)).

\subsection{Population and Sample The Study}

Population used was Albino female mice aged 16-20 weeks with an average weight of 20-30 grams, which is obtained from the Laboratory of Maros Balitbang Veterinary. Mice were maintained for 1 week under standard conditions (temperature $28 \pm 20 \mathrm{C}$ ), humidity $50 \pm 100 \mathrm{C}$ and the room lights with a cycle of 12 hours lit and extinguished 12 hours. The samples were tissue of the skin (epidermis + dermis) is taken from the back of mice albino female who met the inclusion criteria. The research sample were randomized into 6 groups.

\subsection{Data Collection Method}

Each mice that had qualified research (inclusion criteria) taken photos the for initial data and during the study. After adaptation for 1 week, 30 mice were divided into 6 groups. Mencit body weight was measured every week. Subjects were divided into 6 groups: 1) Group 1 untreated (5 mice), 2) Group 2 only UVB irradiated with a power of $450 \mathrm{~mJ}$ (5 mice) three times a week for 12 weeks. 3) Group 3 is the back skin of mice (5mice) were given cocoa seed extract topically $100 \mathrm{ppm}$ and UVB irradiated three times a week for 12 weeks. 4) Group 4 is skin the back of mice ( 5 mice) were given cocoa seed extract topically $200 \mathrm{ppm}$ and UVB irradiated three times a week for 12 weeks. 5) Group 5 is the back skin of mice (5 mice) were given cocoa seed extract topically $400 \mathrm{ppm}$ and UVB irradiated three times a week for 12 weeks. 6) Group 6 is the back skin of mice ( 5 mice) were given cocoa seed extract topically $800 \mathrm{ppm}$ and UVB irradiated three times a week for 12 weeks. The treatment was done for 12 weeks. At week 13, all mice were terminated by way of ether given as an anesthetic later in the cervical dislocation performed excisional biopsy of the skin middle 
back of a $2 \times 2 \mathrm{~cm}$ and then stored in a solution of $\mathrm{NaCl} 0.9 \%$ inspection of MDA.

\subsection{Data Analysis Techniques}

Data collected is all the data obtained from the results of studies subsequent edited, tabulations and entered into a computer program, an analysis of descriptive and analytic. Of the five groups will be tested normality with $\mathrm{T}$ test (Mann-WhitneyTest). If the distribution of normal data, then tested by ANOVA followed by Post Hoc test.

\section{Results}

Experimental animal studies have been conducted with experimental design pure to determine the protective effects of cocoa extract on levels of malondialdehyde in albino mice by exposure to UVB. The study was conducted at the Laboratory Animal for Intervention UVB and Cocoa Extract, Laboratory of Microbiology, Faculty of Medicine, University of Hasanuddin Makassar as the immunohistochemical examination.

The study was conducted on 30 female mice were divided into six treatment groups, group 1 back skin of mice ( 5 mice) were given cocoa seed extract topically $100 \mathrm{ppm}$ every day and UVB irradiated three times a week, two groups of mice back skin (5 mice) were given extracts of cocoa beans topically 200 ppm every day and then irradiated UVB three times a week, Group 3 that the back skin of mice ( 5 mice) were given extracts of cocoa beans topically 400 ppm every day and then irradiated UVB three times a week, Group 4, namely leather backs of mice (5 mice) given extracts of cocoa beans every day topically $800 \mathrm{ppm}$ and UVB irradiated three times a week, Group 5 is the back skin of mice ( 5 mice) only UVB irradiated with a power of $450 \mathrm{~mJ}$ three times a week. Group 6 in the form of 5 mice without treatment. All treatments carried out over 12 weeks. Data analysis was performed using SPSS version 22. The statistical method used is the Kolmogorov-Smirnov test to assess data distribution MDA and ANOVA test to compare the mean (mean) MDA between groups of experimental mice, which then performed comparisons between groups using LSD post-hoc test (least significant difference). The test results significant if $\mathrm{p}<0.05$.

Comparison of the mean MDA by group showed difference in a significant the mean levels of MDA in groups, where the levels of MDA significantly highest UVB in the control group (4.87) and lowest in the control group without treatment $(0.86)$.

Table 1. MDA mean comparisons by group.

\begin{tabular}{lllll}
\hline Group & N & Mean & SD & P \\
\hline Cocoa Extract $100 \mathrm{ppm}$ & 5 & 2,37 & 2,40 & \\
Cocoa Extract $200 \mathrm{ppm}$ & 5 & 1,82 & 0,53 & \\
Cocoa Extract $400 \mathrm{ppm}$ & 5 & 3,31 & 1,80 & \multirow{2}{*}{0,014} \\
Cocoa Extract $800 \mathrm{ppm}$ & 5 & 4,17 & 1,42 & \\
Control UVB & 5 & 4,87 & 2,14 & \\
Control negative & 5 & 0,86 & 1,69 & \\
\hline
\end{tabular}

The result of the comparison between groups showed a complete comparison between the experimental group MDA levels. Results obtained are as follows: cocoa extract 100 ppm group had higher levels of MDA significantly lower than the control group UVB. $200 \mathrm{ppm}$ cocoa extract group had higher levels of MDA significantly lower than the control group $800 \mathrm{ppm}$ and UVB group $400 \mathrm{ppm}$ cocoa extract had significantly higher MDA levels than the control group UVB. $800 \mathrm{ppm}$ cocoa extract group had levels of MDA significantly higher than $200 \mathrm{ppm}$ group cocoa extract and controls without treatment. UVB control group had higher levels of MDA significantly higher than the group of cocoa extract $100 \mathrm{ppm}, 200 \mathrm{ppm}$, and controls without treatment. The untreated control group had higher levels of MDA significantly lower than the group of cocoa extract 400 ppm, $800 \mathrm{ppm}$, and control UVB group The untreated control had higher levels of MDA did not differ significantly with MDA levels 100 ppm and 200 ppm group and Control UVB.

\section{Discussion}

The present study shows that cocoa extract $200 \mathrm{ppm}$ give the best effective, decreased levels of MDA thus has a protective effect highest compared with other groups.

The selection of cocoa as protective agent in this study was based on its high flavonoid content. Cocoa contains epicatechin and catechin fraction of flavonoid and procyanidin. The content of easily absorbed of various The mechanism of action studies extract cocoa flavonoid component is still under study, but based on proven research of flavonoids as antioxidants interact with signaling intracellular to get a variety of biological effects. Cocoa therapy prior to induction of stress oxidative preventing DNA damage and increase the activity of antioxidant enzyme glutathione reductase and glutathione peroxidase in cells induced by prooxidant: tetrabuthyl hydroperoxyde. [11] Based on this study also used cacao therapy before induction of pro-oxidants in mice.

Ultraviolet B (UVB) is a spectrum of ultraviolet radiation with a of wave length 290-320 nm, and most effective ultraviolet penetration through the earth and cause damage to human skin. The damage caused by ultraviolet B that is DNA damage cells that form the chromophore. UVB is absorbed into the epidermis andpenetrate to the papillary dermis. Ultraviolet wavelengths most effective cause erythema is 250-290 nm and the effect of decreasing with increasing wavelength. UVB doses can cause damage to the dermis collagen (photoaging) in skin mice of several studies vary greatly. Research in Makassar using UVB and mice as experimental animals, among others, Djawad et al (2008), using the exposure to UVB $343 \mathrm{~mJ} / \mathrm{cm} 2$ three times a week, Waspodo et al (2012), exposure to UVB $343 \mathrm{~mJ} / \mathrm{cm} 2$ three times a week, Adriani et al (2014), exposure to UVB $450 \mathrm{~mJ}$ $/ \mathrm{cm} 2$ three times a week. Referring to the above study, the dose of UVB were used in this study of $450 \mathrm{~mJ} / \mathrm{cm} 2$ three times a week for 12 weeks. [8-10] 


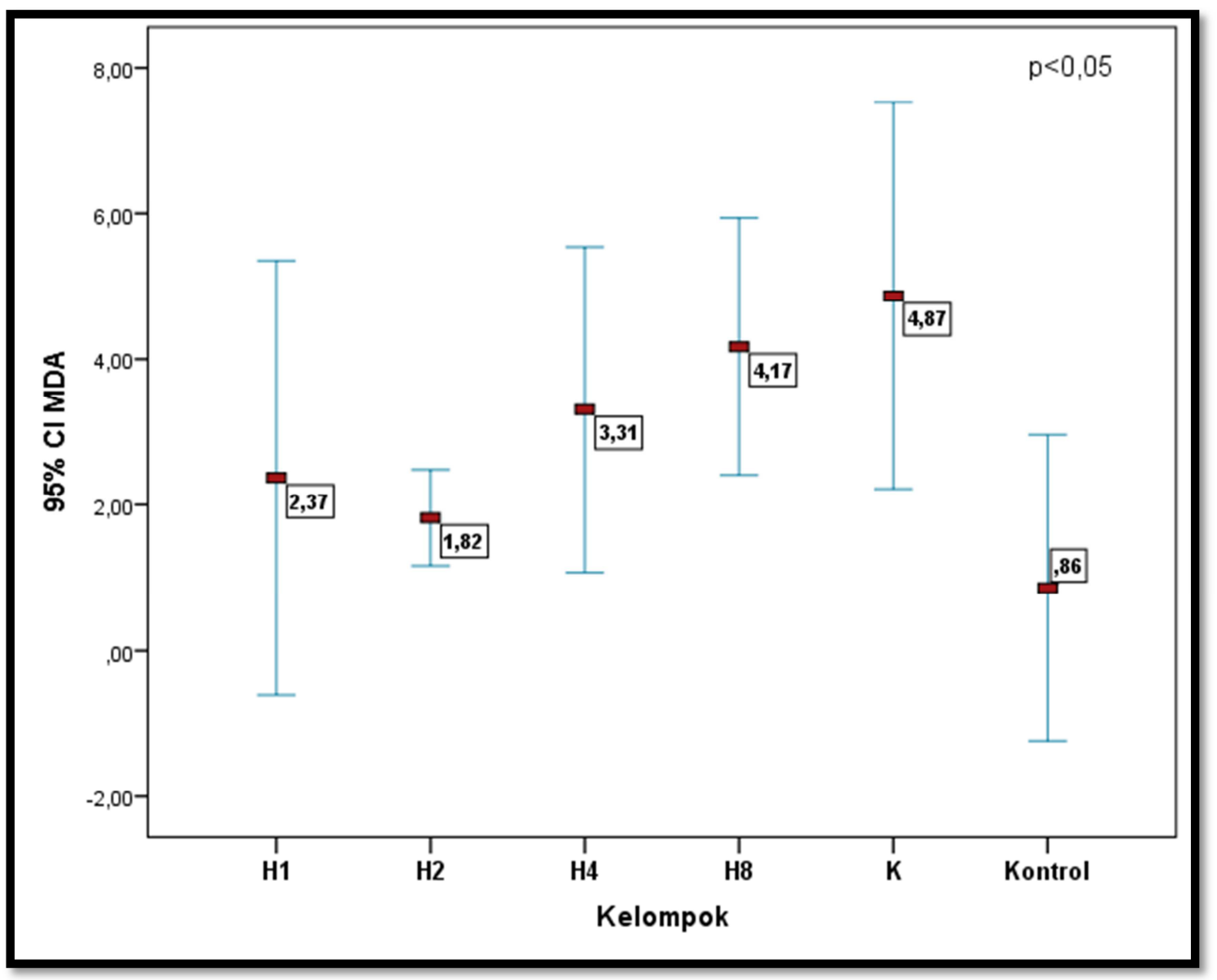

Figure 1. MDA mean comparisons by group.

The results showed MDA, the control group with UVB higher compared with the untreated control group. With the application of $100 \mathrm{ppm}$ extract cocoa decreased levels of MDA and decline with extract cocoa concentration of 200 ppm provides a protective effect on the skin against UVB. Waspodo et al doing experimental research pure cocoa seed extract containing flavonoids in mice exposed to UVB three times a week showed significant at a concentration of 200 ppm associated with improved collagen (TGF-beta and MMP-1). Adriani et al in his research report that cocoa extract concentration of $200 \mathrm{ppm}$ smeared every day and given exposure to UVB three times a week gives the best effect for inhibiting oxidative damage to DNA which is 8OHGD. In connection with this study, that the concentration of 200 ppm cocoa extract applied every day and is exposed to UVB rays provide the best effect with reduced MDA levels that have the highest protective effect compared with other groups. [9, 10]

Increased levels of MDA caused by lipid peroxidation process in which radicals free take electrons present in the lipid in the cell membrane that eventually lead to cell damage. Products produced as a result of lipid peroxidation such as malondialdehyde (MDA), 4-hydroxy-2-noneal (HNE), 4-hydroxy-2-hexenal (4-HHE). MDA is dialdehid compound which is the end product of lipid peroxidation in the body.

In the cocoa extract concentration of $400 \mathrm{ppm}$ and 800 ppm decreased protective effect against UVB. Today the exogenous antioxidants, including polyphenols believed to be a sword" "double edged in stages "redox cell". Some research on exogenous antioxidants showed controversial results, especially when given large doses. It is said that the high concentration of antioxidants also have pro-oxidant activity type, doses of exogenous antioxidants and matrix are factors that determine the balance between beneficial and harmful effects of natural components contained. Exogenous antioxidants in physiological dosage plays an role important in maintaining the redox homeostasis, an important stage in systems a healthy biological. $[5,12]$

Polyphenols are widely considered to be beneficial to human health because they are capable of acting as a free radical Scavenger. But interestingly polyphenols can also act as an agent of ROS at the same time. Therefore, antioxidants should be in sufficient concentration to prevent the accumulation of pro-oxidant. [13] The human body has a mechanism to defend themselves against the harmful effects of ROS are continuously formed.

This is the antioxidant defense system and the stabilization of the reactive components. The fact that some polyphenolic substances have pro-oxidant activity is exciting. Although 
there are few studies on this matter, the fact that stress oxidative can be induced from pro-oxidant agent, either through the formation of ROS or inhibition of antioxidant systems. This can cause oxidative damage to the system of antioxidant biomolecules such as proteins, DNA and lipids and sometimes cells and tissues.

\section{Conclusions and Recommendations}

The authors concluded that there are higher levels of MDA skin mice albino that were not given the extract of cocoa induced UVB rays. There MDA levels were lower in the skin albino mice given the extract of cocoa than $100 \mathrm{ppm}, 200$ ppm, $400 \mathrm{ppm}$ and $800 \mathrm{ppm}$ induced UVB rays. The concentration of the topical application the cocoa extract of best is a dosage of $200 \mathrm{ppm}$. Researchers suggested that topical administration of a dose of seed extract $200 \mathrm{ppm}$ cocoa can be recommended as an antioxidant. Further studies to determine the variation of other cocoa extract effective antioxidant e.g. cocoa extract with a cream base.

\section{References}

[1] Morganelli. (2006). Biography of Chocolate (Paperback). Canada: Crabtree Publishing Co.

[2] Gasser et al. (2008). Cocoa polyphenols and Their Influence on Parameters Involved in ex Vivo Skin Restructuring. Int J Cosmet Sci. 30 (5): 339-45.

[3] McShea et al. (2008). Clinical Benefit and Preservation of flavonols in Dark Chocolate Manufacturing. Nutrition reviews. 66 (11): 630-641.

[4] Othman et al. (2010). Epicatechin Content and Antioxidant
Capacity of Cocoa Beans from Four Different Countries. African Journal of Biotechnology. 9 (7): 1052-1059.

[5] Valko et al. (2007). Free Radicals and Antioxidants in Normal Physiological Functions and Human Disease. Int J Biochem Cell Biol. 39 (1): 44-48.

[6] Godic et al. (2014). The Role of Antioxidants in Skin Cancer Prevention and Treatment. Oxidative Medicine and Cellular Longevity.

[7] Zajac et al. (2008). Food Webs in Long Island Sound: Review, Synthesis and Potential Applications. United States Environmental Protection Agency.

[8] Djawad et al. (2008). Photoprotective effect Curcumin Against Expression CPD, 8-OHdG, Apoptosis and hyperplasia of the epidermis. Makassar: Hasanuddin University.

[9] Waspodo et al. (2012). Role of Cocoa Bean Extract Against Aging Skin After Exposure to Ultraviolet B (Expression of transforming growth factor $\beta$ and matrix Metalloproitenase I. Makassar: Hasanuddin University.

[10] Adriani et al. (2014). Protective Effects of Daily Topical Cocoa Extract to the Expression of $8 \mathrm{OHdG}$ and PCNA on UVB-Exposed Albino Mice. Int J Med Biol Res. 5 (4): 460711 .

[11] Martin et al. (2008). Protection of Human HepG2 Cells Against Oxidative Stress by CocoaPhenolicExtract. J. Agric. Food Chem. 56: 7765-7772.

[12] Bouayed \& Bohn. (2010). Exogenous Antioxidants-Doubleedged Swords in Cellular Redox State: Beneficial Health Effects at physiologic doses Versus deleterious Effects at high doses. Oxid Med Cell Longev. 3 (4): 228-37.

[13] Sies \& Jones (2007). Oxidative Stress. In: Fink G, editors. Encyclopedia of Stress. San Diego: Elsevier. 